
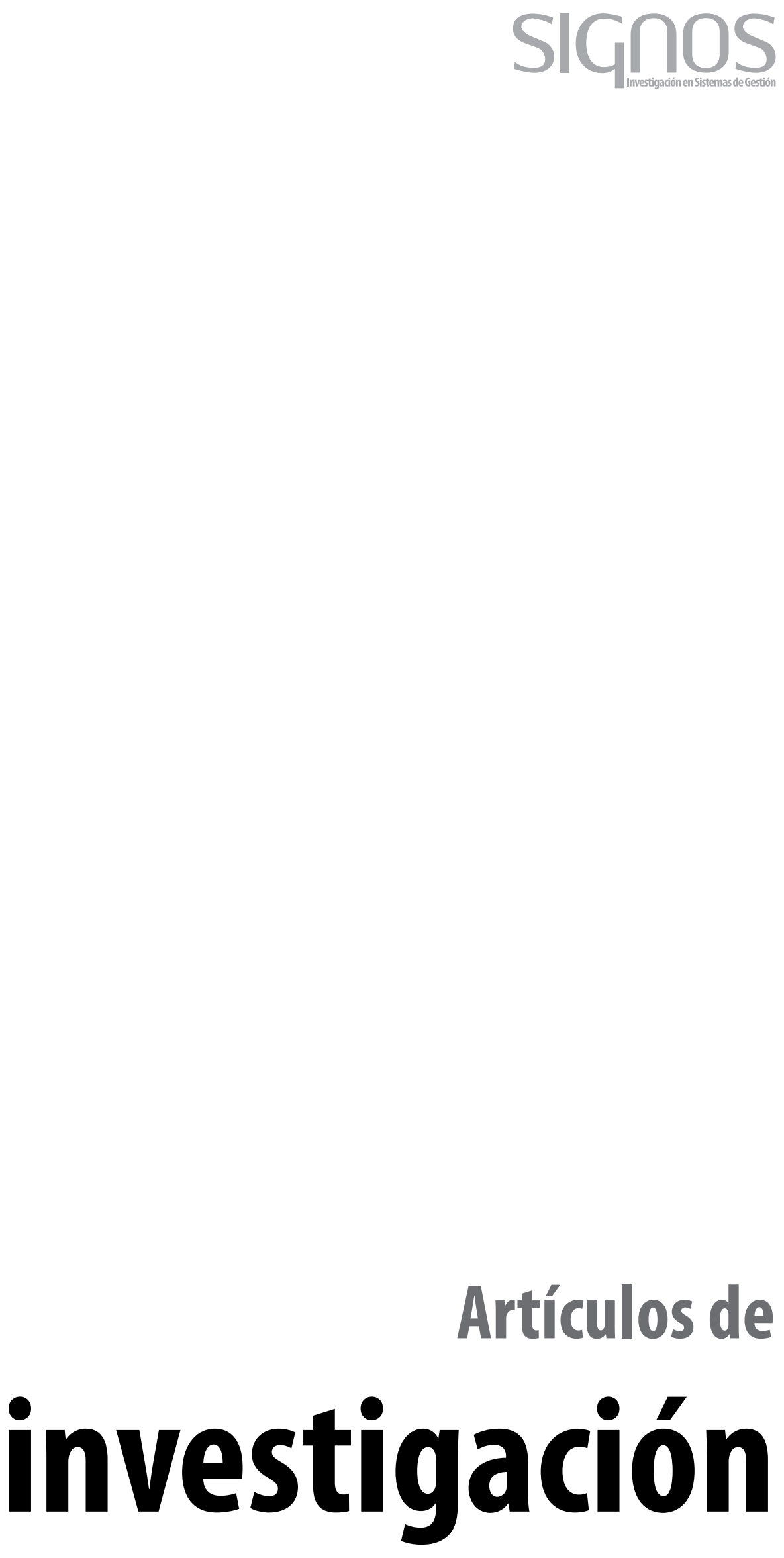


\title{
La actitud de las personas durante la inteǵración del sistema de gestión de la calidad y la acreditación de alta calidad en el programa de Administración Pública de la ESAP*
}

\author{
People's attitudes during the integration process between \\ quality management system and quality accreditation in the \\ Public Administration program at the ESAP
}

Francy Esmeralda Ramire* Castillo **

Diego Fernando Aparicio Fuentes ***

Universidad Santo Tomás e ICONTEC

\section{RESUMEN}

No es común identificar y analizar las manifestaciones resultantes de las actitudes de las personas en la implementación y mantenimiento de los Sistemas de Gestión de la Calidad (SGC). Sin embargo, aunque aparentemente son intangibles, las actitudes influyen en la construcción de la cultura organizacional que favorece u obstaculiza la gestión de la calidad y su integración con otros modelos, como en el caso de la Acreditación de Alta Calidad (AAC) en las Instituciones de Educación Superior (IES).

* Articulo de investigación.

** Profesional especializada Cámara de Comercio de Bogotá. Ingeniera Industrial. Correo electrónico: ing.francyramirez@gmail.com. Autor para correspondencia

*** Docente, Escuela Superior de Educación Pública - ESAP. Arquitecto. Correo electrónico: lemoire23@yahoo.com 
A través de un análisis hermenéutico, el objetivo de esta investigación es interpretar las actitudes de las personas en sus componentes cognitivo, emotivo y conductual en la gestión integral del SGC y la ACC del programa académico de Administración Pública de la Escuela Superior de Administración Pública (ESAP). Para llevar a cabo esta labor se aplicaron instrumentos cualitativos de recolección de información y se pudieron reconocer los patrones culturales a través de categorías deductivas asociadas a la gestión integral y a las actitudes.

En conclusión, se encontró que las actitudes son un elemento fundamental que facilita la gestión integral. Por lo tanto, es conveniente tener en cuenta su incidencia en todas las fases del ciclo de la gestión PHVA, a partir del desarrollo de estrategias y metodologías que permitan orientar las creencias, percepciones, emociones y comportamientos de las personas para favorecer la integración de la gestión.

Palabras clave: gestión integral, sistema de gestión de la calidad, actitudes, cultura organizacional.

\section{ABSTRACT}

In the implementation and maintenance of management systems (QMS) it is not common to identify and analyze manifestations resulting from the attitudes of people. Although attitudes are seemingly intangible, they influence the construction of an organizational culture that favors or hinders quality management, and its integration with other models, such as the High Quality Accreditation (AAC) in Higher Education Institutions (HEI).

Based on a hermeneutical analysis, the purpose of this research aims to interpret people's attitudes considering their cognitive, emotional and behavioral components in the management of the QMS and the AAC, in the Public Administration program at Escuela de Administración Puiblica (ESAP). Using qualitative data collection instruments, we were able to recognize cultural patterns through deductive categories associated with the integrated management and attitudes.

As a result, it was found that attitudes are a fundamental element that facilitates the integrated management system and, therefore, its impact on all phases of the PDCA management cycle should be considered. Thus, developing strategies and methodologies to guide people's beliefs, perceptions, emotions and behavior of individuals can promote the management integrity.

Keywords: Integral management, quality management system, attitudes, organizational culture.

\section{INTRODUCCIÓN}

Las Instituciones de Educación Superior (IES), al igual que otras organizaciones, en su interés de aumentar el logro de sus propósitos, y satisfacer las necesidades de sus usuarios, han optado por implementar un sistema de gestión de la calidad (SGC) siguiendo el modelo ISO 9001. Del mismo modo, el Estado Colombiano, en respuesta a su deber de velar por la calidad de la Educación en Colombia, ha desarrollado mecanismos de control tales como la Ley 1188 de $2008^{1}$ "Por la cual se regula el Registro Calificado de Programas de Educación Superior y se dictan otras disposiciones" e igualmente su Decreto reglamentario el 1295 de $2010^{2}$

1 Ley 1188. Por la cual se regula el registro calificado de los programas de educación superior. Diario Oficial de Colombia. 25 de abril de 2008.

2 Decreto 1295. Por el cual se reglamenta el registro calificado de que trata la Ley 1188 de 2008. Diario oficial de Colombia. 20 de abril de 2010. 
"Por el cual se reglamenta el Registro Calificado de que trata la Ley 1188 de 2008 y la oferta y desarrollo de Programas Académicos de Educación Superior".

Como reconocimiento a las IES que voluntariamente lo soliciten, se otorga la Acreditación de Alta Calidad (AAC) a los Programas de Educación Superior y a las Instituciones que cumplan con unos estándares determinados por el Consejo Nacional de Acreditación de la República de Colombia (CNA).

Sin embargo, no existe seguridad que al implementar el SGC según el modelo ISO 9001:2008 se facilite la obtención de la acreditación, ni que la implementación de los dos modelos y la gestión para la misma se genere de forma integral en las IES.

Ahora bien, durante la implementación de modelos de gestión en las organizaciones existen diferentes recursos que articulados permiten su implementación. Sin embargo, las personas generan la dinámica y permiten que la implementación se lleve a cabo. Es así como el SGC, según el modelo ISO 9001, define dentro de sus principios que la participación del personal y el liderazgo son claves. Así, se atribuye el logro de los objetivos de la organización a las habilidades de las personas. Un componente que puede modificar estas habilidades es la actitud con la cual las personas abordan los acontecimientos cotidianos de la organización. De igual manera, el Consejo Nacional de Acreditación deja entrever en el siguiente párrafo cómo el trabajo en equipo permite la generación y continuidad del proceso de evaluación:

El trabajo colectivo que implica la realización de la autoevaluación y la legitimación interna de todo el proceso, fortalece visiblemente los lazos que dan identidad a la comunidad universitaria, intensifica los sentimientos de pertenencia y mejora el clima institucional dando mayor sentido al trabajo de cada uno dentro del proyecto colectivo ${ }^{3}$.

La presente investigación analiza en el Programa de Administración Pública de la Escuela Superior de Administración Pública (ESAP): ¿De qué modo la actitud de las personas favorece u obstaculiza la articulación entre el Sistema de Gestión de la Calidad según el modelo de la ISO 9001 y la Acreditación de Alta Calidad de acuerdo con los requisitos del CNA?

En este sentido es importante identificar: ¿Qué conocimientos, emociones y comportamientos tienen las partes involucradas en la implementación del SGC y la AAC, con respecto a la gestión integral en el Programa de Administración Pública de la ESAP?

De este interrogante surge otro, cuya respuesta se obtiene a través del desarrollo de la investigación: ¿Las actitudes de las personas influyen en la Gestión Integral del SGC y la AAC en el programa de Administración Pública de la ESAP?

Así, el objetivo de la investigación es interpretar las actitudes de las personas durante la gestión integral del SGC y la AAC en el programa de Administración Pública de la ESAP. Para ello se acude a las personas participantes en la implementación y el mantenimiento de los modelos de gestión estudiados y se analiza el modo como las actitudes de las personas en cada uno de los componentes cognitivo, emotivo y conductual se manifiestan en la integración del SGC y la AAC. Finalmente, se plantean aportes conceptuales para favorecer la gestión integral, tal como se ilustra en el siguiente mapa conceptual:

3 COLOMBIA. CONSEJO NACIONAL DE ACREDITACIÓN. Lineamientos para la Acreditación de Programas [en línea]. Enero 2013. [citado en 22 de Marzo de 2013]. Disponible en Internet:<http://www.cna.gov.co/1741/articles-186359_pregrado_2013.pdf> 
Figura 1. Mapa conceptual de la investigación

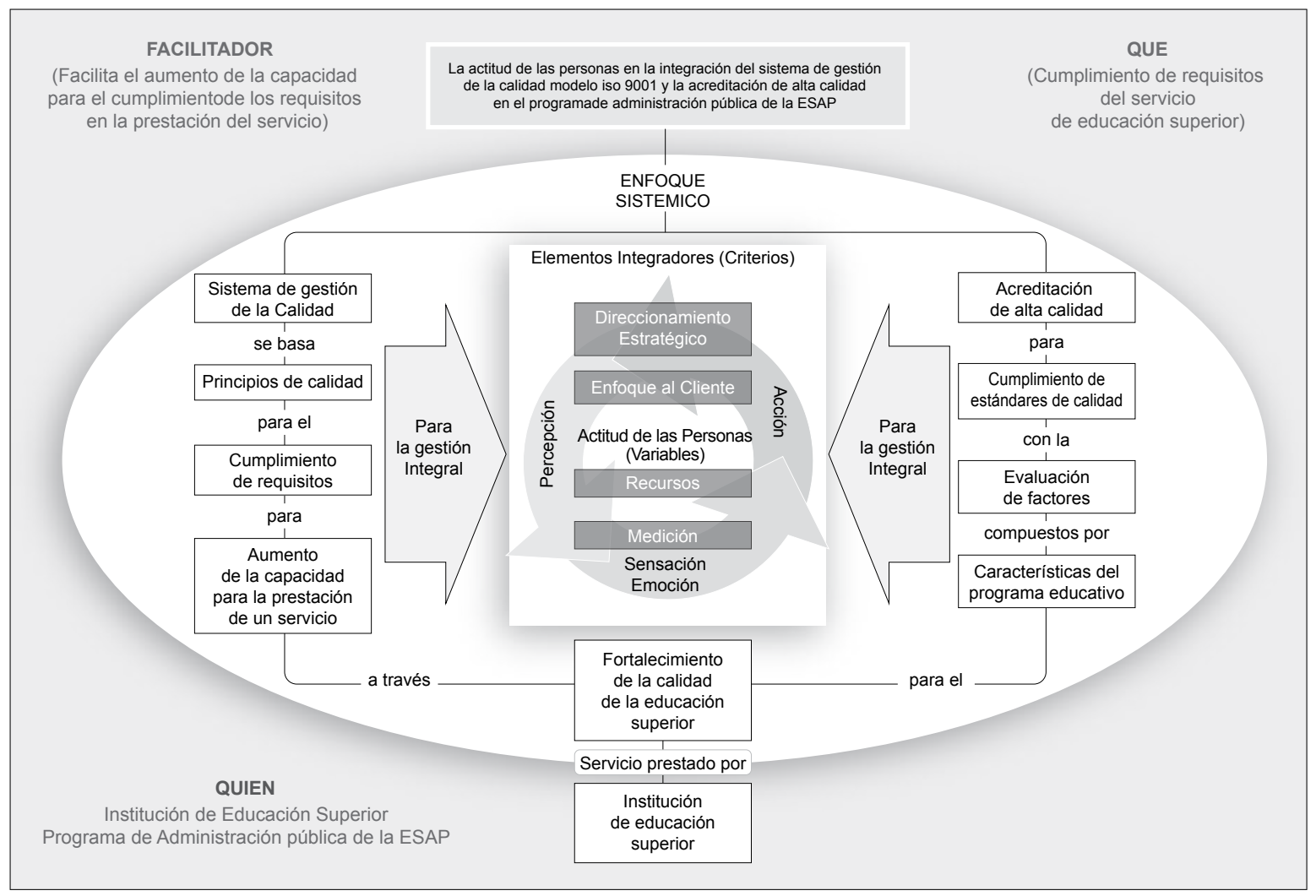

Fuente: elaboración propia

Las actitudes de las personas son un aspecto determinante en la articulación del sistema de gestión de la calidad (SGC) y la acreditación de alta calidad (AAC). Su funcionamiento depende de las personas, las cuales con sus actitudes pueden modificar los resultados, debido a que: "las actitudes son reacciones positivas o negativas hacia objetos, proposiciones abstractas o situaciones" ${ }^{4}$.

Vander añade que una actitud es una tendencia o predisposición adquirida y relativamente duradera a evaluar de determinado modo a una persona, suceso o situación y conlleva a actuar en consonancia con dicha evaluación 5 .

4 PAPALIA, Diane. Psicología. 9 ed. MADRID: McGrawhill., 2004. p. 274

5 VANDER, James. Manual de Psicología Social. BARCELONA: Paidós Ibérica. 1989. p. 26
En la misma línea, Papalia enfoca el concepto de actitud como un comportamiento, forma de pensar o forma de sentir que se aprende y se mantiene a lo largo del tiempo, no es innato ${ }^{6}$. Lo que conduce a determinar que de acuerdo a la forma como una persona aprende acerca de algo y la percepción que adquiere sobre lo aprendido, genera una determinada forma de actuar frente a ello.

Complementando esta premisa Parales-Quenza y Vizcaíno-Gutiérrez expresan:

Desde la perspectiva estructural las actitudes son esquemas que se definen como sistemas de elementos vinculados por una red de relaciones y están organizados de manera jerárquica. Las implicaciones de dicha concepción se relacionan con la idea de estabilidad y cambio; aquellos elementos evocados con más

6 PAPALIA, Op. cit., p. 274 
frecuencia y centrales en la organización tienden a ser más estables y resistentes al cambio ${ }^{7}$.

Las actitudes tienen unas características que se constituyeron en la base teórica para el desarrollo de la presente investigación, a éstas se refiere Rokeach:

1. Conjunto organizado de convicciones o creencias (componente cognitivo): las actitudes suelen presentarse como un conjunto sistemático de creencias, valores, conocimientos, expectativas, etc., que está organizado y cuyos componentes tienen una congruencia y consistencia entre sí.

2. Predisposición o tendencia a responder (componente conductual) de un modo determinado: es una de las características más importantes de la actitud.Aunque no exista una implicación directa entre la actitud y la conducta, normalmente una actitud positiva/negativa hacia algo implica un comportamiento congruente (consistente) con la actitud subyacente.

3. Predisposición favorable o desfavorable hacia el objeto de la actitud: La actitud tiene como componente afectivo-emocional (sentimientos positivos/negativos), por lo que la actitud va acompañada de carga afectiva.

4. Carácter estable y permanente. La estabilidad indica que las actitudes son un conjunto consistente de creencias y actos. Esto no implica que no puedan cambiar, por el contrario, pueden crecer, deteriorarse o desaparecer por factores externos o internos.

5. Las actitudes son aprendidas: se adquieren, principalmente, por procesos de socialización, aunque parece que -en principio- su modo de aprendizaje guarda caracteres específicos frente a otro tipo de aprendizajes. Intervienen en este aprendizaje factores ambientales, sociales y familiares, los medios de comunicación, grupos, la personalidad.

6. Las actitudes desempeñan un papel dinamizador en el conocimiento y en la enseñanza:

7 PARALES-QUENZA, Carlos y VIZCAÍNO-GUTIERREZ, Milcíades. La relación entre actitudes y representaciones sociales elementos para una integración conceptual. [citado en 4 de abril de

2014]. Disponible en http://www.redalyc.org/articulo.oa?id=80539210 se suele tender a conocer aquello hacia lo que se tiene una actitud positiva y a no prestar atención a los objetos, situaciones, o personas asociadas a elementos negativos.

7. Las actitudes son transferibles. Se pueden generalizar y transferir en diferentes situaciones y de diversos modos ${ }^{8}$.

\section{METODOLOGÍA}

Esta investigación busca interpretar la realidad de las personas involucradas en los procesos de implementación de los modelos de gestión de calidad en estudio y la comprensión de sus actitudes frente a la integración de dichos modelos. Para ello se cuenta con el punto de vista de los participantes en su propio contexto a través del método cualitativo, el cual es definido por BonillaCastro y Rodríguez así:

Por tanto, el método cualitativo no parte de supuestos derivados teóricamente, sino que busca conceptualizar sobre la realidad con base en el comportamiento, los conocimientos, las actitudes y los valores que guían el comportamiento de las personas estudiadas. El proceso de investigación cualitativa explora de manera sistemática los conocimientos y valores que comparten los individuos en un determinado contexto espacial y temporal. Esto implica que no aborda la situación empírica con hipótesis deducidas conceptualmente, sino que de manera inductiva pasa del dato observado a identificar los parámetros normativos de comportamiento, que son aceptados por individuos en contextos específicos históricamente determinados ${ }^{9}$.

8 ROKEACH, Milton. Psicología Social. Citado por SÁNCHEZ, Sebastián y MESA, María. Actitudes hacia la tolerancia y la cooperación en ambientes multiculturales. Evaluación e intervención educativa en un contexto concreto. GRANADA: Editorial Universidad de Granada,1998. p. 25-26. colección EIRENE. ISBN: 84-338-2513-5.

9 BONILla-CASTRO, Elssy y RODRIGUEZ, Penélope. Más allá del dilema de los métodos. La investigación en ciencias sociales . BOGOTÁ: Grupo Editorial Norma, 2005. p. 86. 
El proceso de investigación se fundamenta en tres momentos que incluyen siete etapas. De acuerdo a lo expuesto por Bonilla-Castro y Rodríguez, los tres períodos son:

La definición de la situación/problema que abarca la exploración de la situación, el diseño propiamente dicho y la preparación del trabajo de campo.
El trabajo de campo que corresponde al período de recolección y organización de los datos.

La identificación de patrones culturales que organizan la situación y que comprende tres fases fundamentales: el análisis, la interpretación y la conceptualización inductiva ${ }^{10}$.

En la siguiente figura se pueden apreciar los momentos de investigación junto con las etapas desarrolladas.

Figura 2. Esquema metodológico de la investigación

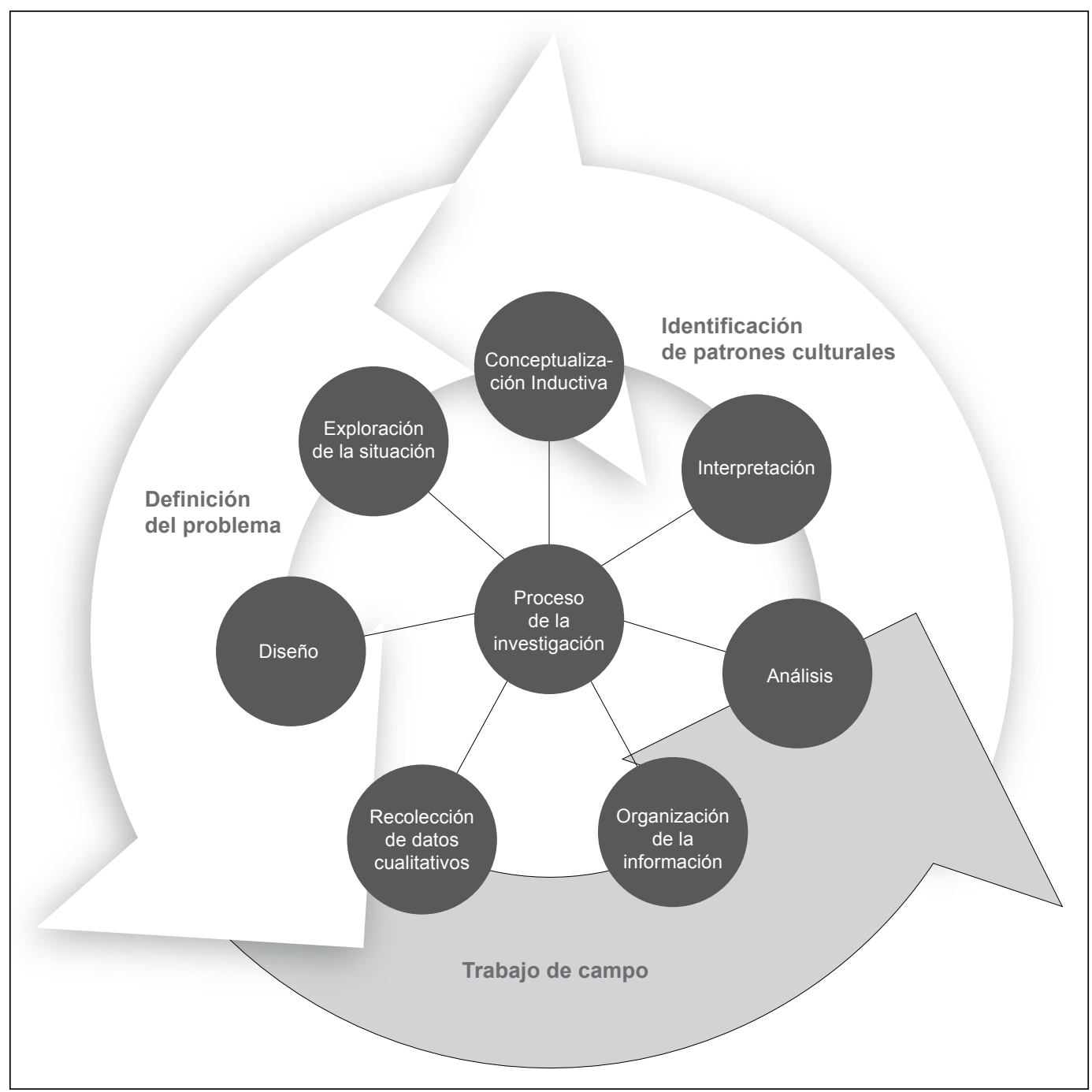

Fuente: diseño proceso de investigación cualitativa.

Elsy Bonilla-Castro y Penélope Rodríguez, en Más allá del dilema de los métodos.

El desarrollo de cada una de estas siete etapas se lleva a cabo de la siguiente manera:

10 BONILLA Y RODRIGUEZ. Op. Cit ., p. 75. 
MOMENTO 1: DEFINICIÓN DEL PROBLEMA.

- Exploración de la situación: en un primer paso se revisa el estado del arte de la investigación que arroja tres estudios previos de la integración del SGC bajo el modelo ISO 9001 y la AAC, en el marco de la Maestría de Calidad y Gestión Integral. Se escoge la investigación de Claudia Pérez denominada "Análisis de interpretación de los conceptos entre la norma ISO 9001:2000 y los estándares de calidad del CNA". A partir de este estudio se valida la integración de los dos modelos, teniendo en cuenta que los lineamientos de la Acreditación de Alta Calidad cambiaron en la organización de las características y factores a partir de la modificación realizada en enero de 2013 (posterior a la fecha de la investigación mencionada anteriormente).

- Diseño: se definen los objetivos y el alcance de la investigación. Se diseñan los instrumentos de medición para la recolección de datos cualitativos, así como las estrategias y los actores (o participantes) para realizar dicha labor.

\section{MOMENTO 2: TRABAJO DE CAMPO.}

- Recolección de datos cualitativos: para la recolección se usan entrevistas informales conversacionales que sirven para indagar acerca de los momentos en los que la integración se da de forma parcial o cuando esta no se da. Finalmente se hace un grupo focal que busca encontrar cómo las actitudes de las personas pueden interferir en el logro de la integración.

\section{MOMENTO 3: IDENTIFICACIÓN DE PARÁMETROS CULTURALES.}

- Organización de la información, análisis e interpretación de los datos cualitativos. Esta etapa inicia con la identificación de las categorías y subcategorías deductivas partiendo de la Gestión Integral, tal como lo ilustra la siguiente figura.

Figura 3. Relación de subcategorías de la gestión integral entre los dos modelos.

\begin{tabular}{|c|l|l|}
\hline Subcategorías de la gestión Integral & Capítulos de la Norma ISO 9001.2008 & $\begin{array}{c}\text { Factores de la acreditación } \\
\text { de alta calidad 2013 }\end{array}$ \\
\hline Direccionamiento Estratégico & Requisitos Generales & $\begin{array}{l}\text { Factor } 1 \text { Misión Proyecto Institucional } \\
\text { y de programa }\end{array}$ \\
\hline Enfoque al Cliente & Responsabilidad de la dirección & Factor 2 Estudiantes \\
\hline Recursos & Recursos & Factor 3 Profesores \\
\hline Prestación del servicio & Seguimiento y medición & Factor 4 Procesos Académicos \\
\hline Seguimiento y medición en subcategoría transversal & $\begin{array}{l}\text { Factor } 5 \text { Visibilidad nacional } \\
\text { e internacional }\end{array}$ \\
\hline & $\begin{array}{l}\text { Factor } 6 \text { Investigación innovación y creación } \\
\text { artística y cultural }\end{array}$ \\
\hline & Factor 7 Bienestar Institucional \\
\hline
\end{tabular}

Fuente: elaboración propia. 
- Con el objetivo de facilitar el análisis de la información se recurre al software ATLAS TI de la siguiente manera:

- Después de haber sido transcritos de un archivo de audio a un archivo de Excel se especifica la participación de cada uno de los actores en forma textual.

- Paralelamente, se procede a la realización del mapa conceptual base para ser utilizado en el análisis e interpretación

Figura 4. Esquema metodológico de la investigación

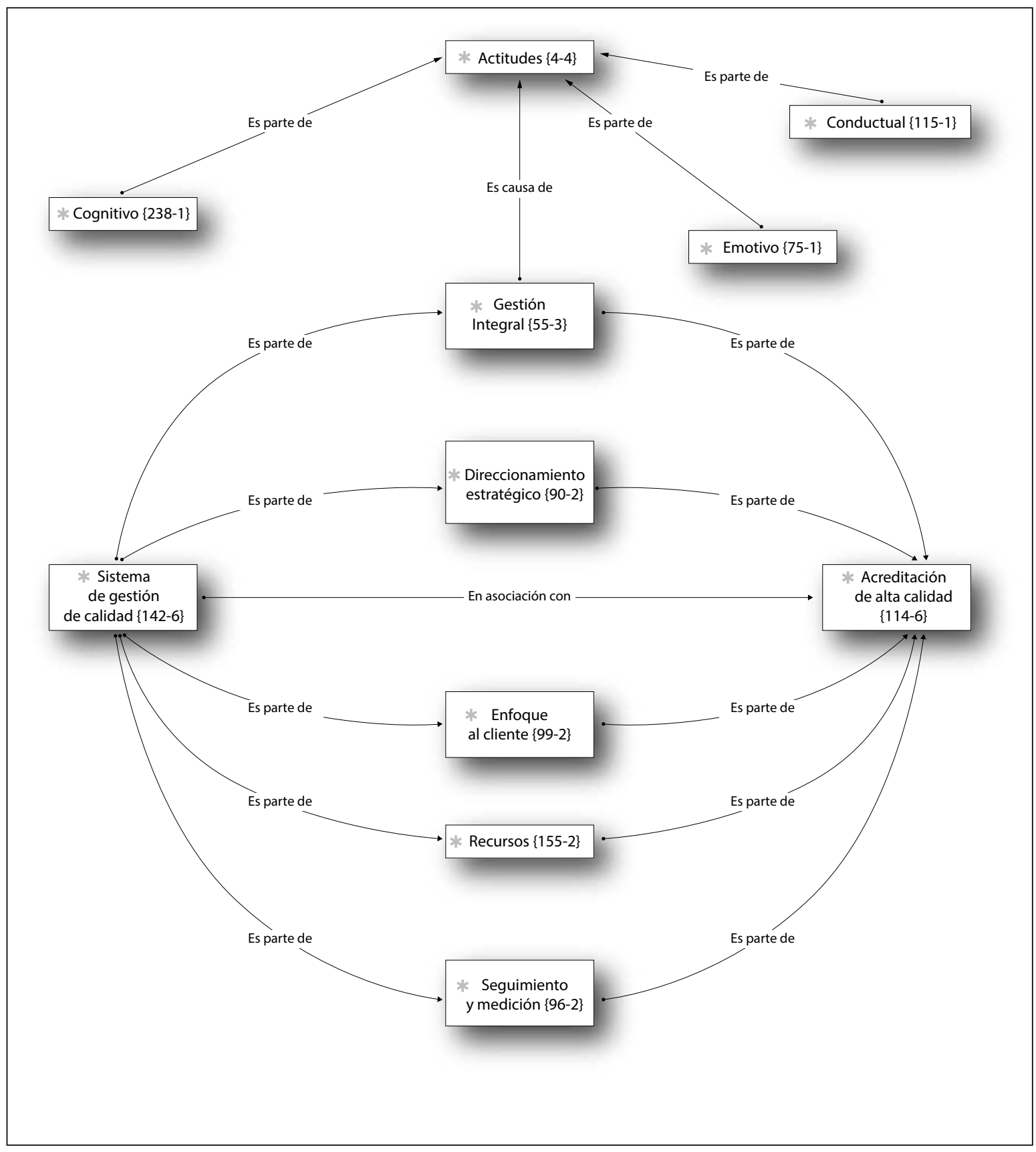

Fuente: elaboración propia. 
Tomando cada uno de los archivos individuales por actor (26 documentos), se identifican en los documentos las citas que tienen relación con las categorías y/o subcategorías deductivas. En la tabla 1 de resultados se organiza de mayor a menor el número de citas relacionadas con cada categoría o subcategoría.

Tabla 1. Codificación inicial de citas

\begin{tabular}{|l|c|}
\hline $\begin{array}{c}\text { CATEGORÍA 0 SUBCATEGORÍA } \\
\text { (CÓDIGOS EN ATLAS TI) }\end{array}$ & No. DE CITAS RELACIONADAS \\
\hline Cognitivo & 238 \\
\hline Recursos & 155 \\
\hline Sistema de gestión de calidad & 142 \\
\hline Conducta & 115 \\
\hline Acreditación de alta calidad & 114 \\
\hline Enfoque al cliente & 99 \\
\hline Seguimiento y medición & 96 \\
\hline Direccionamiento estratégico & 90 \\
\hline Emotivo & 75 \\
\hline Gestión integral & 55 \\
\hline Actitudes & 4 \\
\hline Total & 1183 \\
\hline
\end{tabular}

Fuente: elaboración propia

A partir de la categorización de las unidades de análisis se crean unos nuevos supercódigos. Para ello se extraen aquellas que se relacionen de manera múltiple con las categorías de Acreditación de Alta Calidad, Sistema de Gestión de Calidad y Gestión integral y con las subcategorías de Gestión Integral: direccionamiento estratégico, enfoque al cliente, recursos, seguimiento y medición; y con las subcategorías de las actitudes: cognitivo, emotivo y conductual. De este modo se aumentó la agrupación de la información, quedando de esta manera 385 citas (tabla 2).

Tabla 2. Supercódigos segunda etapa de agrupamiento.

\begin{tabular}{|c|c|c|c|c|c|c|c|c|}
\cline { 2 - 9 } \multicolumn{1}{c|}{} & $\begin{array}{c}\text { DIRECCIONA- } \\
\text { MIENTO } \\
\text { ESTRATÉGICO }\end{array}$ & $\begin{array}{c}\text { ENFOQUE } \\
\text { AL CLIENTE }\end{array}$ & RECURSOS & $\begin{array}{c}\text { SEGUIMIENTO } \\
\text { Y MEDICIÓN }\end{array}$ & COGNITIVO & EMOTIVO & CONDUCTUAL & TOTAL \\
\hline $\begin{array}{c}\text { SISTEMA } \\
\text { DE GESTIÓN DE } \\
\text { CALIDAD }\end{array}$ & 12 & 24 & 27 & 15 & 77 & 10 & 7 & 172 \\
\hline $\begin{array}{c}\text { ACREDITACIÓN } \\
\text { DE ALTA CALIDAD }\end{array}$ & 6 & 13 & 44 & 17 & 49 & 17 & 10 & 156 \\
\hline $\begin{array}{c}\text { GESTIÓN } \\
\text { INTEGRAL }\end{array}$ & 6 & 3 & 9 & 4 & 24 & 3 & 8 & 57 \\
\hline TOTAL & 24 & 40 & 80 & 36 & 150 & 30 & 25 & 385 \\
\hline
\end{tabular}

Fuente: elaboración propia

En la última etapa, antes de la interpretación, se crean unos nuevos supercódigos que agrupan aún más los datos para facilitar la interpretación de los mismos. 
Tabla 3. Supercódigos última relación

\begin{tabular}{|c|c|c|c|c|c|}
\cline { 2 - 6 } \multicolumn{1}{c|}{} & $\begin{array}{c}\text { DIRECCIONAMIENTO } \\
\text { ESTRATÉGICO }\end{array}$ & $\begin{array}{c}\text { ENFOQUE } \\
\text { AL CLIENTE }\end{array}$ & RECURSOS & $\begin{array}{c}\text { SEGUIMIENTO } \\
\text { Y MEDICIÓN }\end{array}$ & TOTAL \\
\hline SISTEMA DE GESTIÓN DE CALIDAD Y COGNITIVO & 3 & 9 & 14 & 5 & 31 \\
\hline ACREDITACIÓN DE ALTA CALIDAD Y EMOTIVO & 0 & 0 & 1 & 1 & 2 \\
\hline SISTEMA DEGESTIÓN DE CALIDAD Y CONDUCTUAL & 1 & 4 & 3 & 2 & 10 \\
\hline ACREDITACIÓN DE ALTA CALIDAD Y COGNITIVO & 0 & 1 & 18 & 5 & 24 \\
\hline ACREDITACIÓN DE ALTA CALIDAD Y EMOTIVO & 1 & 1 & 5 & 3 & 10 \\
\hline ACREDITACIÓN DE ALTA CALIDAD Y CONDUCTUAL & 0 & 1 & 7 & 0 & 8 \\
\hline TOTAL & 5 & 16 & 48 & 16 & 85 \\
\hline
\end{tabular}

Fuente: elaboración propia

Considerando estos supercódigos - y que sus respectivas citas se relacionan directamente con el objeto de estudio -, se toma la decisión de realizar la interpretación con estas 85 citas.

De esta fase se obtiene como resultado la matriz de interpretación y aportes conceptuales.

\section{Conceptualización inductiva}

Siguiendo lo establecido por Hurtado y Toro la inducción permite pasar de los hechos particulares a los principios generales. Consiste en partir de la observación de múltiples hechos o fenómenos para luego clasificarlos y llegar a establecer las relaciones o puntos de conexión entre ellos, pudiendo concluir una teoría ${ }^{11}$. En esta etapa a partir de los fenómenos interpretados se encuentran los aportes conceptuales.

En la validación de la categorización participan tres expertos a quienes se les informa, a través de un documento, acerca de la formulación del problema, los objetivos y la metodología, así como los criterios con los cuales fueron analizadas cada una de las categorías. Ellos hacen una reflexión de dicha

11 HURTADO LEÓN, Ivan y TORO GARRIDO, Josefina. Paradigmas y Métodos de Investigación en Tiempos de Cambio. CARACAS: Editorial CEC,SA, 2007. p.64. categorización e interpretación, emitiendo su concepto con respecto a los criterios de validez mencionados anteriormente.

\section{RESULTADOS Y DISCUSION:}

Una vez categorizados los datos, conforme a las categorías y subcategorías anteriormente descritas, se representa la red de relaciones entre las partes que los componen con el fin de explicar los resultados obtenidos. Con referencia a esto Bonilla-Castro y Rodríguez exponen el proceso a seguir:

El proceso puede darse más o menos de la siguiente forma: a) descripción de los hallazgos aislados; b) identificación de relaciones entre las variables; c) formulación de relaciones tentativas entre los fenómenos; d) revisión de los datos de búsqueda de evidencias que corroboren o invaliden los supuestos que guían el trabajo; e) formulación de explicaciones sobre el fenómeno; f) identificación de esquemas teóricos más amplios que contextualicen el patrón cultural identificado. En cualquier caso, el analista debe asegurarse que la evidencia sustenta sus interpretaciones, apoyándose y ejemplificando con las citas textuales consignadas en los datos ${ }^{12}$.

12 BONILLA-CASTRO y RODRIGUEZ.Op. cit., p. 147. 
Descripción de los hallazgos aislados e identificación de relaciones entre variables.

Siguiendo la metodología, se enumeran las actitudes involucradas asociadas a los componentes cognitivo, emotivo y conductual, y se relaciona la cita en la cual se encuentra su presencia. Para los elementos perdurables

Tabla 4. Componente Cognitivo

\begin{tabular}{|c|c|c|}
\hline No & ACTITUD & $\begin{array}{c}\text { CITA (UNIDAD } \\
\text { HERMENÉUTICA) }\end{array}$ \\
\hline 1 & Acumulación de poder & 77 \\
\hline 2 & $\begin{array}{l}\text { Aportes a la visión global } \\
\text { de la institución }\end{array}$ & 41 \\
\hline 3 & $\begin{array}{l}\text { Aportes de acuerdo con el rol } \\
\text { en el cual se desempeña }\end{array}$ & 6 \\
\hline 4 & Articulación & 29,16 \\
\hline 5 & Autoevaluación & 76 \\
\hline 6 & Avance & 93 \\
\hline 7 & Claridad de los procesos & 65 \\
\hline 8 & Claridad del propósito & 22 \\
\hline 9 & $\begin{array}{l}\text { Coherencia y concordancia } \\
\text { entre todos los elementos } \\
\text { del direccionamiento estratégico }\end{array}$ & 39 \\
\hline 10 & Comunicación con precisión & 1 \\
\hline 11 & Cumplimiento & $14,27,85$ \\
\hline 12 & Desarticulación & $33,34,94$ \\
\hline 13 & Desequilibrio & 26 \\
\hline 14 & Desinformación & 30 \\
\hline 15 & Disminución de la calidad & $58,59,60$ \\
\hline 16 & $\begin{array}{l}\text { La definición de responsabilidades } \\
\text { influye en la eficiencia }\end{array}$ & 64 \\
\hline 17 & $\begin{array}{l}\text { El propósito de los sistemas de } \\
\text { eficiencia }\end{array}$ & 84 \\
\hline 18 & $\begin{array}{l}\text { La importancia de fundamentar los } \\
\text { procesos para que sean entendidos }\end{array}$ & 76 \\
\hline 19 & $\begin{array}{l}\text { Aprovechar la experiencia de los } \\
\text { docentes y de las personas }\end{array}$ & 2,75 \\
\hline 20 & Cada persona tiene fortalezas & 5 \\
\hline 21 & Necesidad, generación de cultura & $22,32,71$ \\
\hline 22 & Gestión fraccionada & 54 \\
\hline 23 & $\begin{array}{l}\text { La política de calidad es una } \\
\text { herraienta dinamizadora }\end{array}$ & 36 \\
\hline
\end{tabular}

Fuente: elaboración propia establecidos por Rokeach, se discriminan en negrilla las actitudes favorables y se sombrean aquellas recurrentes que se evidencian en más de tres citas.

En el componente Cognitivo siguiendo los lineamientos Rokeach, se identifican 23 creencias, valores, conocimientos y expectativas.

Tabla 4. Componente Emotivo

\begin{tabular}{|c|c|c|}
\hline No & ACTITUD & $\begin{array}{c}\text { CITA (UNIDAD } \\
\text { HERMENÉUTICA) }\end{array}$ \\
\hline 1 & Admiración & 40 \\
\hline 2 & Agrado & $22,25,69,72,75,80,92$ \\
\hline 3 & Angustia & 64 \\
\hline 4 & Anhelo & $28,31,96,97$ \\
\hline 5 & Animadversión & 85 \\
\hline 6 & Decepción & 51 \\
\hline 7 & Descontento & 82 \\
\hline 8 & Desilusión & 15 \\
\hline 9 & Disgusto & 66,96 \\
\hline 10 & Dolor & 12 \\
\hline 11 & Enojo & 13 \\
\hline 12 & Entusiasmo & $57,78,93$ \\
\hline 13 & Esperanza & 61 \\
\hline 14 & Expectativa & 39,75 \\
\hline 15 & Frustración & 63 \\
\hline 16 & Gusto & 2,20 \\
\hline 17 & Impotencia & 55 \\
\hline 18 & Inconformidad & $4,26,41$ \\
\hline 19 & Interés por trabajar en las personas & 23 \\
\hline 20 & Ironía & 55 \\
\hline 21 & $\begin{array}{l}\text { Necesidad de reconocer y valorar } \\
\text { los aportes individuales }\end{array}$ & 19 \\
\hline 22 & Pena & 28 \\
\hline 23 & Preocupación & 1 \\
\hline 24 & Repulsión & 33 \\
\hline 25 & Satisfacción y entusiasmo & 57 \\
\hline 26 & Sufrimiento & 25,95 \\
\hline 27 & Tristeza & 59,69 \\
\hline
\end{tabular}

En el componente Emotivo siguiendo los lineamientos Rokeach, se identifican 27 sentimientos positivos o negativos. 
Francy Esmeralda Ramirez Castillo, Diego Fernando Aparicio Fuentes

Tabla 4. Componente Cognitivo

\begin{tabular}{|c|c|c|}
\hline No & ACTITUD & $\begin{array}{c}\text { CITA (UNIDAD } \\
\text { HERMENÉUTICA) }\end{array}$ \\
\hline 1 & Actuar para suplir necesidades & 89 \\
\hline 2 & $\begin{array}{l}\text { Asumir conscientemente } \\
\text { la responsabilidad para el logro } \\
\text { de los propósitos }\end{array}$ & 69 \\
\hline 3 & $\begin{array}{l}\text { Continuar reflexionando en } \\
\text { los por qué }\end{array}$ & 78 \\
\hline 4 & Cumplir con los requisitos & $25,32,26$ \\
\hline 5 & Generar una retroalimentación & 20 \\
\hline 6 & $\begin{array}{l}\text { Hacer promoción al } \\
\text { direccionamiento estratégico }\end{array}$ & 37 \\
\hline 7 & $\begin{array}{l}\text { Integración de las personas aplicado } \\
\text { a los componentes de los sistemas }\end{array}$ & 23 \\
\hline 8 & Integración de actuar para mejorar & $\begin{array}{c}7,23,29,46,69 \\
70,77\end{array}$ \\
\hline 9 & Invita a medir los aportes & 19 \\
\hline 10 & Invitar a una revisión & $58,59,60$ \\
\hline 11 & $\begin{array}{l}\text { Involucrar todos los modelos para } \\
\text { construir la gestión integral }\end{array}$ & 41 \\
\hline 12 & Participación & $17,21,35,51,89$ \\
\hline 13 & Realización de planes de acción & 49 \\
\hline 14 & $\begin{array}{l}\text { Rescatar la riqueza } \\
\text { de conocimientos y experiencia }\end{array}$ & 7 \\
\hline 15 & Responsabilidad & $65,67,69,70,71$ \\
\hline 16 & Trabajar el concepto de integralidad & \\
\hline 17 & $\begin{array}{l}\text { Trabajar en el cumplimiento } \\
\text { de la finalidad de la institución }\end{array}$ & 22 \\
\hline 18 & $\begin{array}{l}\text { Trabajar en la articulación de los } \\
\text { sistemas de información }\end{array}$ & 29 \\
\hline 19 & $\begin{array}{l}\text { Trabajar en la autoevaluación } \\
\text { para que se convierta en una } \\
\text { herramienta para la mejora } \\
\text { continua }\end{array}$ & 73 \\
\hline 20 & $\begin{array}{l}\text { Trabajar en la mejora de } \\
\text { los instrumentos }\end{array}$ & 81 \\
\hline 21 & $\begin{array}{l}\text { Voluntad para recolectar y unificar } \\
\text { la información }\end{array}$ & 10 \\
\hline 22 & $\begin{array}{l}\text { Voluntad para vivir el proceso } \\
\text { de integralidad }\end{array}$ & 43 \\
\hline
\end{tabular}

Fuente: elaboración propia

En el componente Conductual se identifican 22 comportamientos, los cuales Rokeach relaciona con la predisposición o tendencia a responder de un modo determinado 
La siguiente gráfica refleja las relaciones entre variables según lo expresan Bonilla-Castro y Rodríguez, cuyas variables son el total de actitudes por componente, su comparación en relación con los otros y su tendencia positiva o negativa.

Figura 5. Relación entre variables

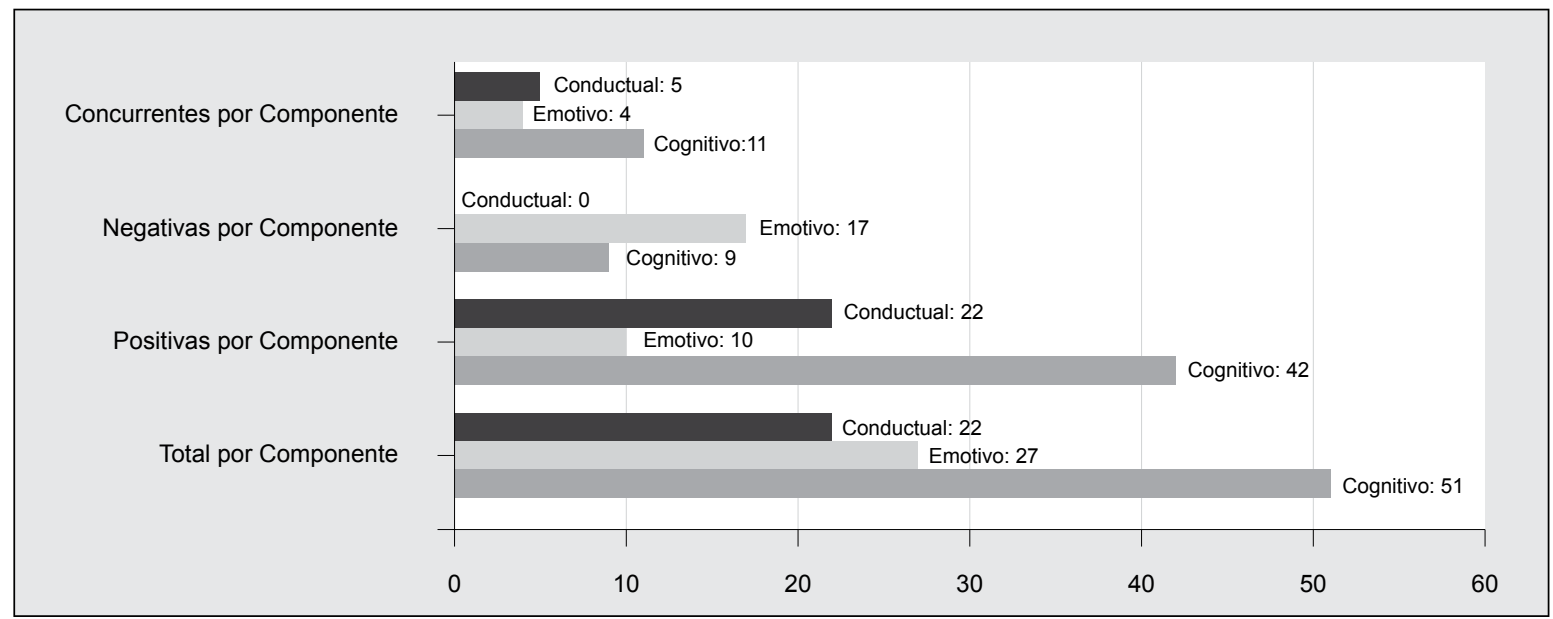

Fuente: elaboración propia

Los datos permiten interpretar la influencia de las actitudes en sus tres componentes, encontrando que lo cognitivo prevalece sobre los otros componentes. El caso de estudio muestra la facilidad que tienen los involucrados para expresar sus convicciones, creencias, valores, conocimientos y expectativas. Al encontrar un mayor número de elementos positivos, se denota la tendencia que el grupo manifiesta hacia los aportes de acuerdo con: el rol de desempeño, contribución al cumplimiento y aprovechamiento de la experiencia de los actores, incluyendo todos los sistemas.

En segundo lugar se encuentra lo emotivo, que hace referencia a los sentimientos positivos o negativos que surgen como respuesta a la experiencia que la persona haya tenido con relación a alguno de los dos modelos
0 a su misma integración. En este aspecto se aprecia la existencia de una tendencia negativa, la cual se manifiesta ante la imposibilidad de poder cumplir lo propuesto: frustración, inconformidad o disgusto (especialmente por la dificultad de dar continuidad a los procesos implementados).

Finalmente, el componente conductual tiene una menor participación debido a que este implica un compromiso para que se manifieste la integración. Sin embargo, en este componente no se aprecian elementos negativos, lo cual se convierte en un insumo para generar cambios. Este componente se evidencia cuando se hace mención a: invitar a la revisión, la participación, la responsabilidad y trabajar en la autoevaluación para que se convierta en una herramienta para la mejora continua. 


\section{Formulación de relaciones tentativas y explicación de los fenómenos con respecto al esquema teórico.}

Para la interpretación de estos resultados se utiliza el esquema teórico del análisis funcional de la actitud expuesto por Pacheco. Este esquema permite igualmente orientar los resultados, identificando relaciones entre los fenómenos de estudio y sustentando los análisis: "Todas las actitudes tienen una función general: Evaluación estimativa. La actitud actúa como premisa 0 alerta ante un objeto que va a percibir. Si no existiera esta función, la persona estaría continuamente evaluando los mismos objetos actitudinales. Por tanto tiene un valor adaptativo" ${ }^{13}$.

Esta evaluación estimativa está presente en aquellas actitudes en las cuales se expresa una toma de decisión, como por ejemplo: la importancia de la información que va a trasmitirse, la inclusión de todos los sistemas y los actores, la intención de actuar para mejorar, el cumplimiento con los requisitos, mantener la continuidad en los sistemas de gestión.

Así mismo las actitudes pueden cumplir las siguientes funciones:

a) Función de valor de orientación

De todos los objetos actitudinales que percibimos sólo prestamos atención a los que nos permiten alcanzar resultados positivos o negativos (en cuanto a evaluación)...

...No todas las actitudes tienen esta función orientadora (de prestar atención) sino que solo se activan actitudes más accesibles ${ }^{14}$.
Entre las actitudes identificadas se encuentran tendencias orientadoras como: la disminución de la calidad, invitación a una revisión, intención de actuar para mejorar, importancia de cumplir unos requisitos. Todo lo anterior es un llamado a mejorar la calidad del servicio educativo ofrecido, lo cual permite al responsable de mantener los sistemas de gestión utilizar estas orientaciones para lograr la integración de los modelos de estudio, partiendo de la necesidad del mejoramiento de gestión.

La siguiente función expuesta por Pacheco es: "b) Función de valoración instrumental. Ocurre cuando se utiliza la actitud para alcanzar un fin, de tal forma que se evalúa de forma positiva al objeto actitudinal, si la persona piensa que va a resultarle gratificante 0 va a conseguir un objetivo y se evalúa negativamente en caso contrario" ${ }^{15}$. En este caso, las actitudes instrumentales que serían apalancadoras para la integración de los modelos establecidos se refleja: mantener la continuidad y la necesidad de generación de cultura. Con la presencia de estas actitudes las personas sienten que los esfuerzos no se perderán al momento de crear mecanismos que generan la cultura organizacional para la gestión integral.

Continuando con lo expuesto por Pacheco: "c) Funciones de expresión de valores: La cumplen aquellas actitudes cuyo fin es poder expresar o conseguir ciertos valores. La actitud actúa como medio para alcanzarlos" ${ }^{16}$. Dentro de las actitudes identificadas claramente se puede apreciar que la responsabilidad, el liderazgo y el compromiso pueden fundamentar los principios de la organización orientando el quehacer de las personas involucradas en la gestión integral de los modelos de estudio en la IES.

13 PACHECO, Francisco. Actitudes. En: Eúphoros. 2002, no. 5, p.173-186

15 Ibid., p.182.

14 Ibid., p.182.

16 Ibid., p.182 
Igualmente se presenta: "d) Funciones de separación: La persona adopta una actitud determinada para repudiar y mantener alejadas a las personas de otro grupo" ${ }^{17}$. En este caso se encuentra la expresión común: "en el momento de la foto nos acomodamos", la cual refleja sentimientos de repulsión asociados a la situación, pues se espera que las personas no actúen de esta manera, sino que se viva realmente el sistema. Según Pacheco:

Funciones ideológicas hegemónica

Su base es un conflicto ostensible entre varios grupos sociales. El conflicto surge de la división social en la que un grupo dominante se impone ante un grupo dominado. El grupo dominado elabora un conjunto de explicaciones ideológicas porque apelan al bien común, al interés social, y con hegemónicas porque el grupo dominante tiene los medios suficientes para imponer estas explicaciones y poder adoptar esta actitud ${ }^{18}$.

Finalmente, un marcado acento de ideología hegemónica se encuentra en las citas de: acumulación de poder (cita 77) en la cual existe un agrado por la destrucción de esas hegemonías. También la cita 55 exterioriza un sentimiento de ironía sobre la superioridad que se percibe en los docentes y la desilusión ante la imposibilidad de mejorar las competencias. Estas situaciones deben recibir una atención especial para evitar obstáculos en la integración de los Sistemas.

Aportes conceptuales a partir de la contextualización de patrones culturales.

Una vez identificadas y analizadas las actitudes se realizan los aportes conceptuales a partir de los fenómenos interpretados y los patrones culturales con el fin de evidenciar la relación que existe entre ellos.

17 Ibid., p.182

18 Ibid., p.182
En la realización de la investigación aparecieron otras subcategorías: las inductivas, que permiten determinar conceptualmente los aportes que podrán conducir la implementación de modelos de gestión o la integración de los mismos.

\section{Categoría inductiva: cumplimiento de requisitos.}

Para desarrollar actitudes de las personas que favorezcan la gestión integral es necesario generar estrategias que motiven a las personas a conocer y a entender los requisitos del sistema. Lo anterior permitiría que se desarrollaran comportamientos que incluyeran el control de los mismos y de las especificaciones del servicio educativo.

\section{Categoría Inductiva: procesos.}

Para la gestión integral es fundamental el desarrollo de actividades que fomenten la conciencia del impacto de los resultados individuales sobre los colectivos y la interrelación de los procesos - sin importar si son estratégicos, misionales o de apoyo, todos tienen igual importancia -, evitando de esta manera que se generen sentimientos de frustración, inconformidad y desmotivación hacia la mejora. Lo anterior podría fomentar un comportamiento de cooperación y de unidad.

\section{Categoría Inductiva: generación de cultura}

Para instaurar una cultura que soporte la gestión integral es necesario trabajar en las creencias, sentimientos y comportamientos de todos los involucrados, fortaleciendo los principios y valores hacia la mística del servicio, lo cual se reflejará en actuaciones que repercuten tanto al interior como al exterior de la institución. De esta manera no se limita la gestión al cumplimiento de funciones o procedimientos estipulados. Al conocer el propósito real de la institución se tiene conciencia de los resultados esperados, 
dando respuesta efectiva a las necesidades de los usuarios. Este ejercicio debe ser continuo, replicarse a todos los niveles de la organización y acompañarse de la recolección de las percepciones y el conocimiento de las expectativas que las personas tengan sobre los modelos de gestión a integrar.

\section{Categoría Inductiva: propósito de la institución}

El Direccionamiento Estratégico permite saber hacia dónde dirigir la Institución de Educación Superior, sin embargo, para que exista una gestión integral es importante, desde el punto de vista cognitivo, trabajar en determinar los lineamientos comunes, y a través de la comunicación, facilitar la interiorización de los propósitos de la institución. El no trabajar de esta manera podría generar que las personas se sientan excluídas. En este sentido, se limitarían las oportunidades para generar motivación y entusiasmo, lo que daría pie a comportamientos consecuentes con estas emociones.

\section{Categoría Inductiva: intereses compartidos}

Para que exista una gestión integral - desde lo cognitivo - se hace necesario que las personas tengan claridad acerca de sus comportamientos para generar convicción sobre una visión global y sistémica. Así, los sentimientos de pertenencia permiten alinear los esfuerzos para llegar más allá de la elaboración de los documentos, procedimientos y registros, para enfocarse en el logro de los resultados y optimizar los recursos utilizados.

\section{Categorías inductivas: responsabilidad, autoridad y liderazgo}

En la gestión integral es fundamental establecer los propósitos de la institución, definir las responsabilidades de todos los involucrados en su cumplimiento, y ejecutarlas como está previsto. Cuando la gestión integral articula las actividades individuales con los propósitos colectivos desde el componente cognitivo de las actitudes se manifiesta la responsabilidad en lo conductual.

\section{Categoría Inductiva: control}

El trabajo de fortalecer las actitudes se encuentra en todas las etapas del ciclo PHVA. Entre las actividades de seguimiento, especialmente en las auditorías, debe existir conciencia y preocupación por contribuir en la mejora continua, y no enfocarse únicamente en evidenciar aspectos negativos, pues esta actitud se traduciría en percepciones de obligación en los auditados, y no en reflexión para la mejora de los procesos.

\section{Categoría Inductiva: documentación e información}

En la construcción de la integración es fundamental que los documentos de los sistemas de gestión alimenten el conocimiento de los involucrados, de modo que la conducta sea coherente con la integración propuesta. El hecho de que no estén plasmadas las actividades en los documentos de los sistemas implica que la gente no se sienta identificada con los mismos y generen una desmotivación, lo cual impide que éstos puedan ser parte de la mejora continua de las IES, ya que no se va a realizar el seguimiento pertinente para tal fin.

\section{CONCLUSIONES}

A través del análisis e interpretación de cada una de las citas extractadas de las entrevistas informales conversacionales y del grupo focal, así como a la luz de las categorías y subcategorías establecidas con respecto a la manifestación de las actitudes de las personas en sus componentes cognitivo, emotivo y conductual en la gestión integral, se puede concluir que: 
- La concurrencia en las convicciones o creencias de las personas se manifiestan de manera sistemática, respondiendo a una organización consecuente con la interacción de los actores, manteniendo una relación lógica, coherente y consistente, que permitió identificarlas y clasificarlas conforme a las categorías de la investigación. Como en el caso de la importancia que tiene para los actores la información, la forma cómo ésta se transmite a todos los niveles, incluyendo los usuarios, denota para ellos la calidad del servicio en todas las partes interesadas.

- Según las expresiones de los participantes se evidencia la existencia de carga afectiva que se manifiesta de manera positiva o negativa influyendo en la gestión integral, en respuesta al conjunto de convicciones y creencias, como también en las experiencias recogidas durante los procesos de implementación y mantenimiento de los dos modelos de gestión. Con respecto a esta característica hubo manifestaciones concurrentes de desilusión y sufrimiento por el hecho de sentir que se esperaba más del Sistema de Gestión y de su integración con otros sistemas en la ESAP, debido a que con la certificación y la acreditación se pensó que se había logrado superar el nivel precedente de desorganización. Sin embargo ¿̇ómo se refleja lo anterior en el servicio al usuario, en los sistemas de información, en la calidad académica?. Adicionalmente, se despierta un anhelo por una transformación para que estos ejercicios puedan generar una dinámica diferente y se puedan cumplir las expectativas iniciales antes de la implementación de dichos modelos.

- Es evidente, en el análisis de las actitudes, que las convicciones, creencias y la carga afectiva presentan respuestas positivas o negativas, las cuales se contrastan con el componente conductual. Este componente, a diferencia de los dos primeros, refleja la intención y la voluntad de actuar, orientada positivamente hacia el mejoramiento de la gestión. $\mathrm{Al}$ respecto se evidencia que existe una intención de recolectar y unificar la información para mejorar el desempeño y gestión de los procesos, como también expresiones de voluntad frente a la integración de estos u otros modelos. Sin embargo, no ha sido representativa su existencia, pues este componente requiere de un compromiso difícil de exteriorizar por los actores, donde el componente conductual tiene una participación inferior a los demás componentes.

- A lo largo de las entrevistas se encontraron elementos en las actitudes que fueron permanentes, demostrando que mientras no existan eventos dinamizadores del cambio, éstas se mantienen estables y permanecen en el tiempo. Sin embargo, la sola realización de los ejercicios de recolección de información en la presente investigación, generó reflexiones que posibilitan la transformación de las convicciones o creencias, así como de la carga emocional y comportamientos existentes. Este es el caso de la motivación por trabajar de forma integral desde el punto de vista administrativo y académico, realizando procesos de inclusión y participación.

- Las transformaciones de las actitudes que los actores experimentaron durante la presente investigación demuestran su papel dinamizador en el conocimiento y en la enseñanza. Lo anterior indica que en la medida que las personas se sientan identificadas con los propósitos de la institución se afecta el desempeño de la gestión integral.

- Durante la investigación los participantes hicieron referencia a la importancia del liderazgo. Por esta razón, en los procesos de implementación y mantenimiento, es esencial reconocer a las personas que poseen esa competencia, propiciando la participación 
activa de todos, transfiriendo esas actitudes positivas a otros. Así se demuestra que las actitudes, aun siendo individuales, pueden transferirse dada la condición de sociabilidad del ser humano y el grado de influencia que posea el individuo.

Con respecto a los aportes conceptuales como derivación de la identificación, análisis e interpretación de las actitudes, es importante llegar a la definición de acciones a partir de los patrones culturales encontrados debido a que las implicaciones de dicha concepción se relacionan con la idea de estabilidad y cambio. Aquellos elementos evocados con más frecuencia y centrales en la organización tienden a ser más estables y resistentes al cambio. De ahí la prioridad por definir acciones que permitan que se rompa esa red de relaciones entre los tres componentes de la actitud, y que se aprovechen aquellas que pueden favorecer la gestión integral, de modo que puedan ser trabajadas las que la obstaculizan, con el fin de facilitar los procesos de implementación y mantenimiento de la misma.

Teniendo en cuenta lo anterior, esta investigación evidencia la necesidad de incluir la gestión del recurso humano en la gestión integral dando respuesta al problema planteado de la siguiente manera:

- Desde el ideal de formación de las personas:

- Incluyendo metodologías y herramientas de aprendizaje que exploren las actitudes durante todo el ciclo PHVA en los planes de capacitación (Cognitivo), motivación y bienestar (Emotivo), evaluación y seguimiento (Conductual) centrado en las personas.

- En los procesos gana y gana:

- Considerar los diferentes factores individuales, intralaborales y extralaborales que deben ser evaluados al interior de la institución al implementar Sistemas de Gestión Integral.
- Desde la congruencia de los objetivos individuales y los objetivos comunes, estudiar los siguientes aspectos:

- Selección de personal por competencias

- Pertenencia

- Integración

- Trabajo en equipo

- Comunicación

\section{RECOMENDACIONES}

En la actualidad el estudio de las actitudes de las personas y sus aportes a la gestión integral no cuenta con muchos referentes, por esta razón continuar investigando sobre la temática facilitará el desarrollo de metodologías que permitan impactar de manera positiva a todos los involucrados. Con el presente estudio se abre la puerta a nuevas indagaciones como las siguientes:

- Identificar y analizar las actitudes de las personas y su influencia en cada uno de los pasos del ciclo PHVA.

- La influencia de las actitudes en la toma de conciencia.

- Estudio de los impactos sobre las actitudes en los procesos gana y gana.

- Desarrollar herramientas metodológicas para convertirlas en facilitadoras de la gestión integral.

- Estudios para fortalecer las competencias del implementador y del auditor a través del estudio de las actitudes.

También se recomienda incluir en los Programas de Educación Superior contenidos relacionados con la gestión integral y al manejo de las actitudes. 
Con respecto a la aplicación de metodologías de investigación cualitativa de corte hermenéutico, se resalta su importancia al generar nuevos espacios de aproximación a la realidad de las personas involucradas en la organización que implementan y mantienen sistemas de gestión orientados hacia la integración. Se recomienda continuar fortaleciendo las competencias de los investigadores y facilitar herramientas para el análisis de datos cualitativos.

\section{BIBLIOGRAFÍA}

COLOMBIA. CONSEJO NACIONAL DE ACREDITACIÓN. Lineamientos para la Acreditación de Programas [en línea]. Enero 2013. [citado 20 de Agosto de 2013]. Disponible en Internet: <http://www.cna.gov.co/1741/articles-186359 _pregrado_2013.pdf

BONILLA-CASTRO, Elssy y RODRIGUEZ, Penélope. Más allá del dilema de los métodos. La investigación en ciencias sociales. Bogotá: Grupo Editorial Norma, 2005. 220 p.
HURTADO LEÓN, Ivan y TORO GARRIDO, Josefina. Paradigmas y Métodos de Investigación en Tiempos de Cambio. Caracas: Editorial CEC,SA, 2007. $163 \mathrm{p}$

PACHECO, Francisco. Actitudes. En: Eúphoros. 2002, no. 5, p.173-186

PAPALIA, Diane. Psicología. 9 ed. Madrid: McGrawhill., 2004. 420 p.

PARALES-QUENZA, Carlos y VISCAÍNO-GUTIERREZ, Milcíades. Las relaciones entre actitudes y Representaciones sociales: Elementos para una integración conceptual. En: Revista Latinoamericana de Psicología. Mayo-agosto, 2007, vol. 39 no. 2, p. 351-361. ISSN:0120-0534

ROKEACH, Milton. Psicología Social. Citado por SÁNCHEZ, Sebastián y MESA, María. Actitudes hacia la tolerancia y la cooperación en ambientes multiculturales. Evaluación e intervención educativa en un contexto concreto. Granada: Editorial Universidad de Granada,1998. 336 p.

VANDER, James. Manual de Psicología Social. Barcelona: Paidós Ibérica. 1989. 697 p. 